\title{
Formation of heavy element rich giant planets by giant impacts
}

\author{
H. Genda ${ }^{1}$, M. Ikoma ${ }^{2}$, T. Guillot ${ }^{3}$ and S. Ida ${ }^{2}$ \\ ${ }^{1}$ Research Center for the Evolving Earth and Planets, Tokyo Institute of Technology, \\ 2-12-1 Ookayama, Meguro-ku, Tokyo, Japan \\ email: genda@geo.titech.ac.jp \\ ${ }^{2}$ Department of Earth and Planetary Sciences, Tokyo Institute of Technology, \\ 2-12-1 Ookayama, Meguro-ku, Tokyo, Japan \\ ${ }^{3}$ Observatoire de la Côte d'Azur, \\ CNRS UMR 6202, 06304 Nice Cedex 4, France
}

\begin{abstract}
We have performed the smoothed particle hydrodynamic (SPH) simulations of collisions between two gas giant planets. Changes in masses of the ice/rock core and the $\mathrm{H} / \mathrm{He}$ envelope due to the collisions are investigated. The main aim of this study is to constrain the origin and probability of a class of extrasolar hot Jupiters that have much larger cores and/or higher core/envelope mass ratios than those predicted by theories of accretion of gas giant planets. A typical example is HD 149026b. Theoretical models of the interior of HD 149026b (Sato et al. 2005; Fortney et al. 2006; Ikoma et al. 2006) predict that the planet contains a huge core of 50-80 Earth masses relative to the total mass of 110 Earth masses. Our SPH simulations demonstrate that such a gas giant is produced by a collision with an impact velocity of typically more than 2.5 times escape velocity and an impact angle of typically less than 10 degrees, which results in an enormous loss of the envelope gas and complete accretion of both cores.
\end{abstract}

Keywords. planetary systems, formation, hydrodynamics

\section{Introduction}

More than twenty extrasolar planets are known to transit their star. From planetary radius observed by the transit method and planetary mass observed by the radial velocity method, one can determine the densities of the extrasolar planets. The density of the planet informs us about the planetary interior. According to calculation of the interior structure of gas giant planet by Guillot et al. (2006), the amount of the heavy elements (ice/rock core in their model) in nine transiting extrasolar planets have been estimated (see Fig. 1$)$. It is noted that the core mass $\left(M_{\text {core }}\right)$ and core mass ratio $\left(x_{\text {core }}\right)$ in the planets increases with the metallicity of their star $([\mathrm{Fe} / \mathrm{H}])$. This dependency seems to be reasonable, because the star with higher $[\mathrm{Fe} / \mathrm{H}]$ had the protoplanetary disk with enough solid materials to form more heavy element-rich planet. However, as mentioned below, the simple formation theory of gas giant planets cannot fully explain this dependency.

According to the core accretion model for gas giant planet formation, when a solid core grows to a certain critical mass (called the critical core mass), the gaseous envelope around the core begins to contract and gas giant planet forms. Typical critical core mass is estimated to be $\sim 10 M_{E}$ (Mizuno 1980), and upper limit is $\sim 30 M_{E}$ (Ikoma et al. 2006), where $M_{E}$ is the Earth's mass. Assuming that the composition of the gaseous envelope is the same as the composition of its star, we can estimate the total amount of the heavy elements in the planet (i.e., core + heavy element in envelope). The graycolored region in Fig. 1 shows the estimated range of total heavy elements in the gas 

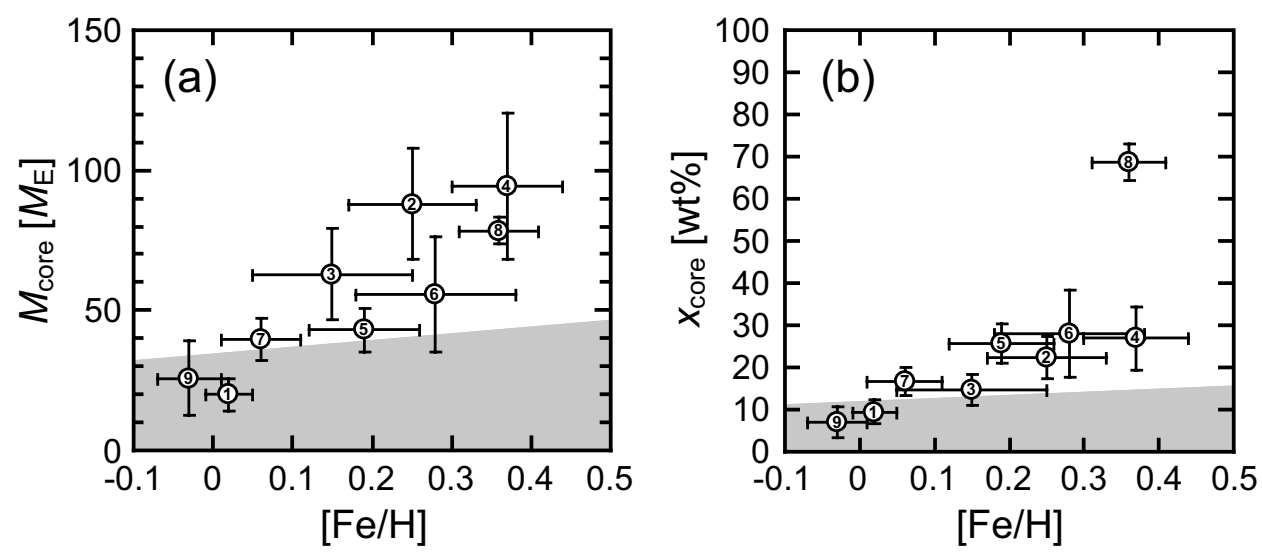

Figure 1. Core mass and core mass ratio in the planets as a function of the metal content of the parent star (figures modified from Guillot et al. 2006). The number in the circle corresponds to each planet; (1) HD209458b, (2) OGLE-TR-56b, (3) OGLE-TR-113, (4) OGLE-TR-132, (5) OGLE-TR-111, (6) OGLE-TR-10, (7) TrES-1, (8) HD149026b, and (9) HD189733b. Horizontal error bars correspond to the $1 \sigma$ errors on the determination of the stellar metallicity $([\mathrm{Fe} / \mathrm{H}])$. Vertical error bars are a consequence of the uncertainties on the measured planetary radii, masses and ages. (a) The core mass $\left(M_{\text {core }}\right)$, which is normalized by the Earth's mass $\left(M_{E}\right)$, increases with $[\mathrm{Fe} / \mathrm{H}]$. (b) The core mass ratio of planet $\left(x_{\text {core }}\right)$ also increases with $[\mathrm{Fe} / \mathrm{H}]$. The gray-colored regions show the theoretically estimated range of total heavy elements in the gas giant planet with a Jupiter mass.

giant planet with a Jupiter mass. The transiting planets around the star with relatively high $[\mathrm{Fe} / \mathrm{H}]$ have too much heavy elements.

Here we examine the collision between two gas giant planets as one of the possible mechanism to make a gas giant planet rich in heavy elements. We show that the collision increases both the core mass and core mass ratio of a planet owing to merging of two cores and erosion of envelopes. We discuss the dependence of collision probability of gas giants on $[\mathrm{Fe} / \mathrm{H}]$ of their star.

\section{Numerical Results}

To perform the simulations of a collision between two gas giant planets, we use the smoothed particle hydrodynamic (SPH) code (e.g., Lucy 1977; Canup 2004). A collision is represented by 20,000 SPH particles, and the mutual gravity between the SPH particles is calculated using the special-purpose computers named micro-GRAPE (Fukushige et al. 2005). The equations of state we used are the Tillotson EOS (Tillotson 1962) with the parameters of ice for the core, and ideal gas with ratio of specific heat 2 or SCVH EOS (Saumon et al. 1995) for the envelope.

We consider the gas giant planet with $30 M_{E}$ of the core and $270 M_{E}$ of the envelope, and perform the simulations of the collision between two same-sized gas giant planets. We show two examples of time series of our simulations in Fig. 2. Panels (a)-1 to (a)-4 show the time sequence of collision with $v_{i m p}=1.5 v_{e s c}$ and $\theta=5^{\circ}$, and panels (b)- 1 to (b) -4 show that of collision with $v_{i m p}=3.0 v_{e s c}$ and $\theta=0^{\circ}$, where $v_{i m p}$ is the impact velocity, $v_{e s c}$ is the two-body escape velocity, and $\theta$ is the impact angle (The collisions for $\theta=0^{\circ}$ and $90^{\circ}$ correspond to head-on and grazing collisions, respectively). In both cases, the cores in two gas giant planets (grey dots) merge. The envelopes (white dots) 

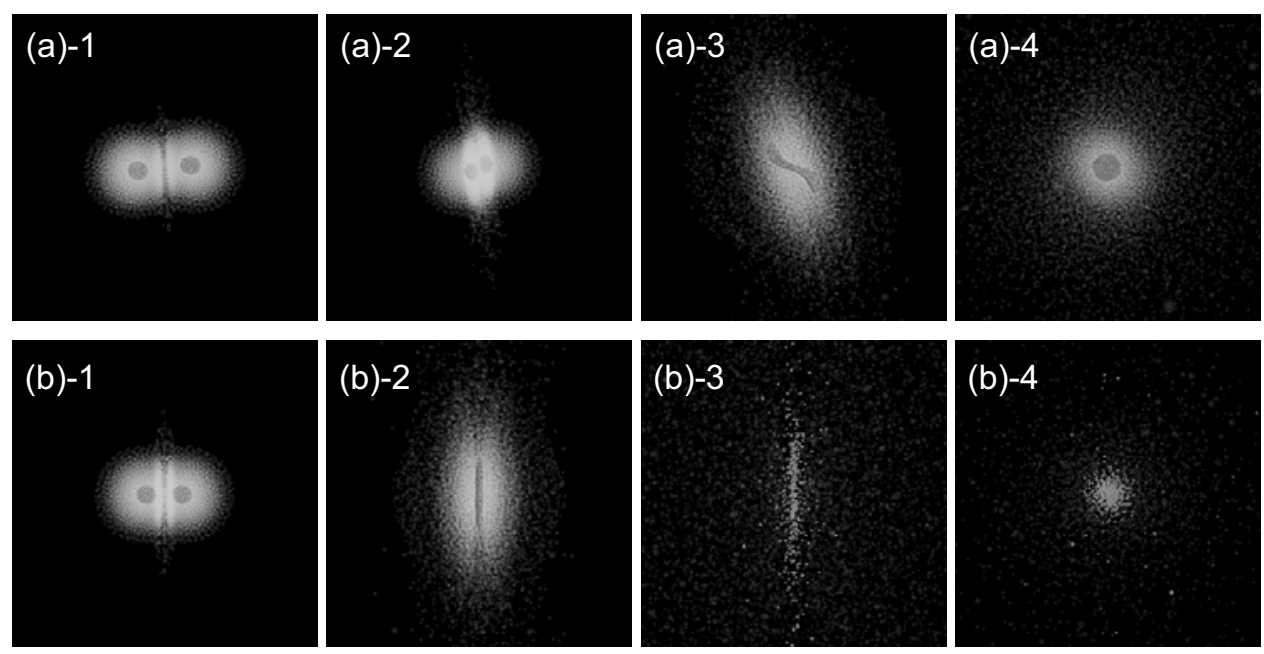

Figure 2. Snapshots of simulations for collisions between two gas giant planets. Panels from (a)-1 to (a)-4 show time sequence of a relatively low velocity collision, and panels from (b)-1 to (b)-4 show that of a relatively high velocity collision. White dots correspond to the $\mathrm{H} / \mathrm{He}$ envelope, and gray dots, icy core.

are more drastically eroded in the case (b) than (a), because the impact velocity in the case (b) is higher than (a). The masses of envelope and core of the largest gravitationally bound aggregate at the end of the simulations ( $\sim 10^{5}$ seconds) are $470 M_{E}$ and $60 M_{E}$ for case (a), respectively, and $80 M_{E}$ and $57 M_{E}$ for case (b). The core mass ratios are (a) $11.3 \mathrm{wt} \%$ and (b) $41.6 \mathrm{wt} \%$. Since $10 \mathrm{wt} \%$ of the initial core mass ratio is considered, the collision makes the core mass ratio increase.

Fig. 3 shows the final mass $\left(M_{1}\right)$ and core mass ratio of the largest gravitationally bound aggregate at the end of the simulations for various impact velocities and impact angles. Collision with higher impact velocity results in higher core mass ratio. To produce a class of heavy element-rich planet such as HD 149026b by a single collision event, the collision with high impact velocity and low impact angle is required.

\section{Discussion}

As can seen from Fig. 1, the planet around the star with higher $[\mathrm{Fe} / \mathrm{H}]$ has higher core mass and higher core mass ratio. To make this dependency by collision between two gas giant planets, the planetary system with higher $[\mathrm{Fe} / \mathrm{H}]$ needs to have higher probability of collisions between gas giant planets. Here, we discuss the collision probability of the planetary system.

It is known that the planetary system with more than three gas giant planets is orbitally unstable. In the case for the planetary system with three gas giant planets around $5 \mathrm{AU}$, one planet is ejected on an hyperbolic trajectory for most cases, and a collision between two gas giant planets occurs for $\sim 5 \%$ of the cases (Marzari \& Weidenschilling 2002). If gas giant planets are located close to the star due to a certain mechanism of inward migration of the planet such as Type II migration, the collision probability rises. This is because the hill radius of the planet decreases with the semi-major axis but physical radius is constant. The collision probability also may increase with the number of gas giants. The extrasolar planets observed by the transit method so far are located close 

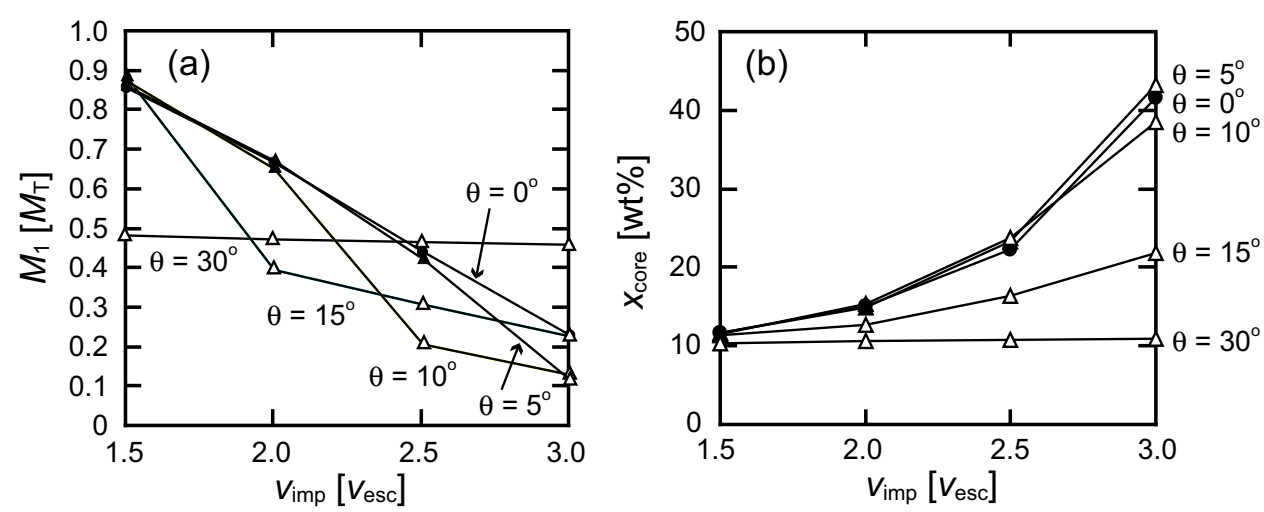

Figure 3. Collisional output for various impact velocities $\left(v_{i m p}\right)$ and impact angles $(\theta)$. (a) Mass of the largest gravitationally bound aggregate $\left(M_{1}\right)$ normalized by total mass of initial two gas giant as a function of $v_{i m p}$ normalized by the two-body escape velocity $\left(v_{e s c}\right)$. (b) Core mass ratio of the largest gravitationally bound aggregate $\left(x_{\text {core }}\right)$ as a function of $v_{i m p}$. Two gas giant planets do not always merge. Filled symbols correspond to merging events, while open symbols hit-and-run events.

to their star. Therefore, the collision probability of the planetary system with transiting planet would be higher than $5 \%$, if more than three gas giant planets initially formed.

According to core accretion model for gas giant planet formation, gas giant planet forms from solid core. Therefore, we can speculate that the star with higher $[\mathrm{Fe} / \mathrm{H}]$ used to have more solid material in protoplanetary disk and more gas giant planets can form. Observation also seems to be consistent with the above speculation, because 3 planetary systems out of $62(\sim 5 \%)$ are multiple planetary systems in the case for $[\mathrm{Fe} / \mathrm{H}]<0$, while 18 out of $138(\sim 13 \%)$ for $[\mathrm{Fe} / \mathrm{H}]>0$. Therefore, the collision probability qualitatively increases with $[\mathrm{Fe} / \mathrm{H}]$ of the star. To make the dependency of core mass and ratio of planet on $[\mathrm{Fe} / \mathrm{H}]$ precisely, we need more investigation about the number of gas giant formed in protoplanetary disk and collision probability for wide range of $[\mathrm{Fe} / \mathrm{H}]$.

\section{References}

Canup, R. M. 2004, Icarus, 168, 433

Fortney, J. J., Saumon, D., Marley, M. S. Lodders, K., \& Freedman, R. S. 2006, ApJ, 642, 495

Fukushige, T., Makino, J., \& Kawai, A. 2005, PASJ, 57, 1009

Guillot, T., Santos, N. C., Pont, F., Iro, N., Melo, C., \& Ribas, I. 2006, A\&̋A, 453, L21

Ikoma, M., Guillot, T., Genda, H., Tanigawa, T., \& Ida, S. 2006, ApJ, 650, 1150

Lucy, L. B. 1977, AJ, 82, 1013

Marzari, F. \& Weidenschilling, S. J. 2002, Icarus, 156, 570

Mizuno, H. 1980, Prog. Theor. Phys., 64, 544

Sato, B., et al. 2005, ApJ, 633, 465

Saumon, D., Chabrier, G., \& Van Horn, H. M. 1995, ApJS, 99, 713

Tillotson, J. H. 1962, Report No. GA-3216, General Atomic, San Diego, California 